\title{
Learning US Assisted Neuraxial Technique during a Pandemic: Case Report
}

\author{
Martin May ${ }^{1 *}$, Galitzine ${ }^{2}$ and Matthews $\mathrm{J}^{2}$ \\ ${ }^{1}$ ST7 Anaesthetic Registrar, Nuffield Department of Anaesthetics, Oxford University Hospitals NHS Foundation Trust, Oxford, UK \\ ${ }^{2}$ Consultant Anaesthetist, Nuffield Department of Anaesthetics, Oxford University Hospitals NHS Foundation Trust, Oxford, UK \\ ${ }^{\star}$ Corresponding author: Martin May, 7 Sherwood Place, Oxford, UK; Email: martin.may1@nhs.net
}

Received: December 29, 2021; Accepted: January 05, 2022; Published: January 12, 2022

\begin{abstract}
We present the case of an anaesthetic registrar learning ultrasound assisted neuraxial anaesthesia during the COVID-19 pandemic. An 81-year-old patient with significant scoliosis required anaesthesia for a revision hip replacement following periprosthetic fracture. Despite the communication difficulties presented by wearing full personal protective equipment to reduce potential transmission of COVID-19 an experienced consultant anaesthetist was able to demonstrate the relevant lumbar spine sonoanatomy to a year six anaesthetic registrar. The registrar was then able to perform a first pass neuraxial procedure in the left lateral position under general anaesthesia. The anaesthetic had good effect and the patient was comfortable post operatively with minimal opiate requirements. The patient was discharged to his home from hospital 14 days later.
\end{abstract}

Keywords: Ultrasound, Spinal anaesthesia, Neuraxial, Case report

\section{Introduction}

Spinal anaesthesia is a common technique for hip and knee replacements and revisions. It is not uncommon to encounter difficulty in finding the intervertebral space using a landmark technique in this population; who tend to be elderly with osteoarthritic changes to the spine, may have scoliosis and may have had previous spinal surgery. Difficult anatomy can lead to multiple passes of the spinal needle with the inherent increased risk and discomfort for the patient. There is evidence that pre-procedural ultrasound imaging can reduce the number of needle passes [1-4]. We report this case of ultrasound imaging guiding a senior anaesthetic trainee to perform a first pass spinal anaesthetic technique on an asleep patient with scoliosis and previous spinal surgery requiring surgery for periprosthetic femoral fracture during the 2020 Covid-19 pandemic.

\section{Case Report}

An 81-year-old male was listed for a right revision hip replacement following periprosthetic fracture during the 2020 Covid-19 pandemic. The patient had well controlled hypertension, long-standing right sided sciatica and scoliosis with an American Society of Anaesthesiologists physical status IIE. He had had previous bilateral hip replacements, lumbar decompression and recent cervical decompression. Preoperative investigations showed a haemoglobin of $107 \mathrm{~g} / \mathrm{L}$; his Covid-19 swab was negative. An MRI report described exaggerated lumbar lordosis and significant scoliosis with moderate to severe canal narrowing at L4/5.

The patient was consented for general anaesthesia with spinal anaesthesia for analgesia. Anaesthesia was commenced in the operating theatre with staff wearing full Personal Protective Equipment (PPE) including long sleeve gown, two pairs of gloves, a visor and an FFP3 mask or equivalent (local hospital policy at this time). Anaesthesia was induced with propofol, fentanyl and rocuronium and was maintained with sevofurane in a mixture of oxygen and nitrous oxide. The patient's trachea was intubated orally using a McGrath video-laryngoscope with a size $8 \mathrm{~mm}$ (ID) standard tracheal tube. Following intubation, a radial arterial line was sited and the patient placed in the left lateral position. A consultant anaesthetist with extensive experience in the use of ultrasound assisted neuraxial techniques scanned the patient's back with a curvilinear probe. Using ultrasound, the consultant demonstrated the sacrum and identified the lumbar intervertebral spaces showing narrow gaps and a longitudinally rotated spine. Spinous process shadow on the ultrasound showed that in the left lateral position the midline was significantly superior to the previous decompression scar (Figure 1).

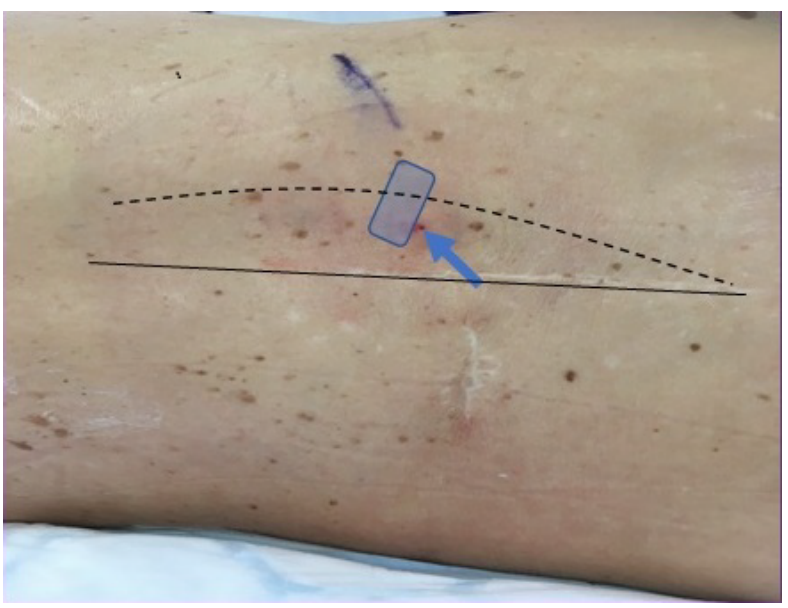

Figure 1: Picture of patient's back showing expected midline (solid line) and actual spinous processes (dashed line) with rectangle indicating pre-procedure curvilinear probe position and arrow indicating entry point and direction of needle. 
The optimal space was identified (L3/4), distance to the laminae was calculated to be $4 \mathrm{~cm}$ allowing the depth of the spinal canal to be estimated at $4-5 \mathrm{~cm}$. Angulation of the probe suggested the needle should be aimed towards the right shoulder to penetrate the dura. The site was marked and the year six anaesthetic trainee donned sterile gown and gloves whilst the site was cleaned with $0.5 \%$ chlorhexidine spray. The trainee was experienced in neuraxial procedures using landmark technique but had no experience using ultrasound imaging of the spine. Despite muffled voices from wearing the FFP3 masks the consultant conveyed the information provided by ultrasound to the trainee. The trainee punctured the dura on the first pass using a needle angle he would not have used otherwise. Local anaesthetic in the form of $0.5 \%$ plain bupivacaine was injected and surgery was allowed to commence. During surgery the patient did not respond to surgical stimulus indicating functioning spinal anaesthetic. Surgery lasted 180 minutes and at the end we performed femoral and lateral cutaneous nerve blocks. The patient stayed in an overnight recovery area where his pain was described as mild and settled with $10 \mathrm{mg}$ oral morphine. The patient was discharged home 14 days after surgery.

\section{Discussion}

Spinal anaesthesia is a common technique for lower limb surgery, but it can be challenging in patients with scoliosis or previous spinal surgery. Technically difficult neuraxial techniques in asleep paralysed patients carry additional risks, especially when performed using landmarks [5]. In the presented case a potentially difficult spinal was rendered straightforward by the pre-procedure use of ultrasound imaging. If not for the serendipity of having a consultant present experienced in the use of ultrasound assisted neuraxial procedures this spinal was likely to require multiple attempts, increased time and may have been impossible for the operating anaesthetist.

Ultrasound has the potential to be an extremely useful tool in performing neuraxial procedures. It can be used to identify a safe level for needle insertion, to identify the midline, to estimate the depth of the spinal canal and show any longitudinal rotation of the spine. This can reduce time to successful dural puncture, improved patient satisfaction and reduced complication rate [6].

Intubation and extubation were identified as aerosol generating procedures and high risk for the spread of Covid-19 leading to a preference for regional anaesthesia over GA where possible $[7,8]$. At the time of this case it was recommended to wear full PPE when conducting general anaesthesia and staff were issued with either an FFP3 mask, a JSP Force 8 or Force 10 mask (JSP, Minster Lovell, UK). These masks provide protection, but they lead to increased communication difficulties. The PPE muffled voices and was uncomfortable to wear for a prolonged period making it difficult to concentrate. Despite this it was possible to impart the relevant information to render a difficult spinal straightforward.

Twenty years ago, central venous access and peripheral nerve blocks were all performed routinely without ultrasound. Many anaesthetists now consider ultrasound guidance to be mandatory in the performance of these procedures and this is reflected in national guidance. We argue that, with the increasing availability of ultrasound, anaesthetists should become more familiar using ultrasound when performing neuraxial techniques. In fact, National Institute for Health and Care Excellence guidance from 2008 advocates the use of ultrasound in locating the epidural space for catheterisation. Despite this the current curriculum from the Royal College of Anaesthetists in the UK does not mention the use of ultrasound guided neuraxial procedures. We suggest that ultrasound guided neuraxial should be taught early in anaesthetic training in order for trainees to develop experience with this skill in patients with 'normal' backs.

\section{Acknowledgements}

This case is from June 2020 and is published with the written consent of the patient.

\section{Conflict of Interests}

No external funding and no competing interests declared.

\section{References}

1. Perlas A, Chaparro LE, Chin KJ (2016) Lumbar Neuraxial Ultrasound for Spinal and Epidural Anesthesia: A Systematic Review and Meta-Analysis. Regional Anesthesia and Pain Medicine 41: 251-260. [crossref]

2. Park SK, Yoo S, Kim WH, Lim YJ, Bahk JH, et al. (2019) Ultrasound-assisted vs. landmark-guided paramedian spinal anaesthesia in the elderly: A randomised controlled trial. European Journal of Anaesthesiology 36: 763-771. [crossref]

3. Park SK, Bae J, Yoo S, Kim WH, Lim YJ, et al. (2020) Ultrasound-Assisted Versus Landmark-Guided Spinal Anesthesia in Patients With Abnormal Spinal Anatomy: A Randomized Controlled Trial. Anesthesia and Analgesia 130: 787-795. [crossref]

4. Kallidaikurichi SK, Iohom G, Loughnane F, Lee PJ (2015) Conventional LandmarkGuided Midline Versus Preprocedure Ultrasound-Guided Paramedian Techniques in Spinal Anesthesia. Anesthesia and Analgesia 121: 1089-1096. [crossref]

5. Drasner K. (2004) Thoracic epidural anesthesia: asleep at the wheal? Anesth Analg 99: 578-9. [crossref]

6. Perlas A (2010). Evidence for the Use of Ultrasound in Neuraxial Blocks. Regional Anesthesia \& Pain Medicine 35: supp.43-46.

7. Young B, Onwochei D, Desai N (2020) Conventional landmark palpation vs. preprocedural ultrasound for neuraxial analgesia and anaesthesia in obstetrics - a systematic review and meta-analysis with trial sequential analyses. Anaesthesia.

8. Uppal V, Sondekoppam RV, Landau R, El-Boghdadly K, Narouze S, et al. (2020) Neuraxial anaesthesia and peripheral nerve blocks during the COVID-19 pandemic: a literature review and practice recommendations. Anaesthesia 75: 1350-1363. [crossref]

Citation:

May M, Galitzine, Matthews J (2022) Learning US Assisted Neuraxial Technique during a Pandemic: Case Report. Cancer Stud Ther J Volume 7(1): 1-2. 